\title{
PERIODIC SOLUTIONS FOR NONLINEAR SYSTEMS WITH MEAN CURVATURE-LIKE OPERATORS
}

\author{
PIERLUIGI BENEVIERI, JOÃO MARCOS DO Ó, AND EVERALDO SOUTO DE MEDEIROS
}

\begin{abstract}
We give an existence result for a periodic boundary value problem involving mean curvaturelike operators. Following a recent work of R. Manásevich and J. Mawhin, we use an approach based on the Leray-Schauder degree.
\end{abstract}

\section{INTRODUCTION}

In [5] (see also [6]) Manásevich and Mawhin proved an existence result for the periodic boundary value problem

$$
\left\{\begin{array}{l}
\left(\phi\left(u^{\prime}\right)\right)^{\prime}=f\left(t, u, u^{\prime}\right) \\
u(0)=u(T) \\
u^{\prime}(0)=u^{\prime}(T)
\end{array}\right.
$$

where $f:[0, T] \times \mathbb{R}^{N} \times \mathbb{R}^{N} \rightarrow \mathbb{R}^{N}$ is Carathéodory and $\phi: \mathbb{R}^{N} \rightarrow \mathbb{R}^{N}$ is a homeomorphism satisfying particular monotonicity conditions which include for instance $p$-Laplacian-like operators. They used a topological method: the properties of $\phi$ and $f$ allowed to apply the Leray-Schauder degree to prove that (1.1) admits a solution (see [5, Theorem 3.1]). In [1], proceeding in the general spirit of ManásevichMawhin's ideas, we proved an existence result for a different scalar problem. We considered in fact the periodic boundary value problem

$$
\left\{\begin{array}{l}
\left(\phi\left(u^{\prime}\right)\right)^{\prime}=f\left(t, u, u^{\prime}\right) \\
u(0)=u(T) \\
u^{\prime}(0)=u^{\prime}(T)
\end{array}\right.
$$

where $f:[0, T] \times \mathbb{R} \times \mathbb{R} \rightarrow \mathbb{R}$ is still a Carathéodory function, but $\phi: \mathbb{R} \rightarrow \mathbb{R}$ is, in [1], an increasing homeomorphism between $\mathbb{R}$ and the open interval $(-1,1)$, with $\phi(0)=0$. The interest in this class of nonlinear operators $u \mapsto\left(\phi\left(u^{\prime}\right)\right)^{\prime}$ is mainly due to the fact that they include the scalar version of the mean curvature operator

$$
u \mapsto \operatorname{div}\left(\frac{\nabla u}{\sqrt{1+|\nabla u|^{2}}}\right) .
$$

which is usually considered in the case when $u$ is a real function defined on an open subset of $\mathbb{R}^{N}$.

In this paper we extend the results obtained in [1] to the $N$-dimensional case. Precisely, we study the problem

$$
\left\{\begin{array}{l}
\left(\phi\left(u^{\prime}\right)\right)^{\prime}=f\left(t, u, u^{\prime}\right) \\
u(0)=u(T) \\
u^{\prime}(0)=u^{\prime}(T)
\end{array}\right.
$$

Date: 16 th November 2005

2000 Mathematics Subject Classification. Primary 34B15; Secondary 47H11.

Key words and phrases. boundary value problem, mean curvature-like operators, periodic solutions, Leray-Schauder degree, homotopy invariance. 
assuming that $f:[0, T] \times \mathbb{R}^{N} \times \mathbb{R}^{N} \rightarrow \mathbb{R}^{N}$ is a Carathéodory function and $\phi: \mathbb{R}^{N} \rightarrow \mathbb{R}^{N}$ is a homeomorphism between $\mathbb{R}^{N}$ and the open ball of $\mathbb{R}^{N}$ with center zero and radius 1 , verifying the following condition:

(H1) $\phi(x)=w(\|x\|) x$, for each $x \in \mathbb{R}^{N}$, where $w:[0,+\infty) \rightarrow[0,+\infty)$ is continuous.

We still follow here the topological approach of Manásevich and Mawhin as we did in [1]. Under further assumptions on $f$, which we specify in the sequel, we apply the Leray-Schauder degree showing (Theorem 3.1 below) that (1.2) admits a solution.

We would stress that the results presented here complement those obtained in [5], since we study a class of operators not included in the setting of problems tackled by Manásevich and Mawhin. In particular, referring to [5], an operator $\phi$ satisfying the above $(\mathrm{H} 1)$ does not verify the assumption $\left(\mathrm{H}_{2}\right)$ in [5] (page $369)$.

The paper is organized as follows. In the next section we consider our problem in the particular case when $f$ is independent of $u$ and $u^{\prime}$. The study of this simplified problem is the first step in the direction of applying the Leray-Schauder degree, as done in Section 3. That section is, in particular, devoted to the main theorem of this work, that is, an existence result for system (1.2). In the last section we present an application of the main theorem to a particular system.

We refer to e.g. [3] or [4] for the definition and the main properties of the Leray-Schauder degree.

Standing notation. In what follows $I$ will denote the closed interval $[0, T]$, with $T$ fixed. In addition, we will put $\mathcal{C}=C\left(I, \mathbb{R}^{N}\right), \mathcal{C}^{1}=C^{1}\left(I, \mathbb{R}^{N}\right), \mathcal{C}_{T, 0}=\{u \in \mathcal{C}: u(0)=u(T)=0\}, \mathcal{C}_{T}^{1}=\left\{u \in \mathcal{C}^{1}: u(0)=\right.$ $\left.u(T), u^{\prime}(0)=u^{\prime}(T)\right\}, L^{1}=L^{1}\left(I, \mathbb{R}^{N}\right)$, and, finally, $L_{m}^{1}=\left\{h \in L^{1}: \int_{0}^{T} h(t) d t=0\right\}$. The norm in $\mathcal{C}$ and $\mathcal{C}_{T, 0}$ is defined by

$$
\|u\|_{0}=\max _{t \in I}\|u(t)\|_{\mathbb{R}^{N}}
$$

the norm in $\mathcal{C}^{1}$ and $\mathcal{C}_{T}^{1}$ by

$$
\|u\|_{1}=\|u\|_{0}+\left\|u^{\prime}\right\|_{0}
$$

and the norm in $L^{1}$ and $L_{m}^{1}$ by

$$
\|h\|_{L^{1}}=\left[\sum_{i=1}^{N} \int_{0}^{T}\left\|h_{i}(t)\right\|^{2} d t\right]^{1 / 2}=\left(\sum_{i=1}^{N}\left\|h_{i}\right\|_{L_{1}}^{2}\right)^{1 / 2} .
$$

Finally, by $\|\cdot\|$, we simply denote the Euclidean norm of an element of $\mathbb{R}^{N}$.

Remark 1.1. By a solution of (1.2) we mean a $C^{1}$ real function $u$ on $[0, T]$, satisfying the boundary conditions, such that $\phi\left(u^{\prime}\right)$ is absolutely continuous and verifies $\left(\phi\left(u^{\prime}\right)\right)^{\prime}=f\left(t, u, u^{\prime}\right)$ a.e. on $[0, T]$.

Remark 1.2. During the redaction of this work we have known that a similar result has been obtained, independently, by Bereanu and Mawhin (see [2]). More precisely, they study the Neumann boundary value problem

$$
\left(\phi\left(u^{\prime}\right)\right)^{\prime}=f\left(t, u, u^{\prime}\right), \quad u^{\prime}(0)=u^{\prime}(T)=0,
$$

where $\phi: \mathbb{R} \rightarrow(-a, a)$ is a homeomorphism such that $\phi(0)=0$ and $f:[0, T] \times \mathbb{R} \times \mathbb{R} \rightarrow \mathbb{R}$ is continuous. Bereanu and Mawhin follow a topological approach based on the Leray-Schauder degree (analogously to [5]), and, to this purpose, they find interesting a priori estimates involving the function $f$ and the operator $\phi$. 


\section{AN AUXILIARY PROBLEM}

Consider the following periodic boundary value problem

$$
\left\{\begin{array}{l}
\left(\phi\left(u^{\prime}\right)\right)^{\prime}=h(t) \\
u(0)=u(T) \\
u^{\prime}(0)=u^{\prime}(T),
\end{array}\right.
$$

where $h$ is in $L_{m}^{1}$ and $\phi$ is a homeomorphism between $\mathbb{R}^{N}$ and the open ball of $\mathbb{R}^{N}$, with center zero and radius 1, verifying condition (H1). The next lemma turns out to be useful in the sequel.

Lemma 2.1. For any $x, y \in \mathbb{R}^{N}$ with $x \neq y$ one has

$$
\langle\phi(x)-\phi(y), x-y\rangle>0 .
$$

Proof. Consider first the particular case when $y=\lambda x$, with $\lambda \geq 0, \lambda \neq 1$ and $x \neq 0$. One has that

$$
\begin{aligned}
\langle\phi(x)-\phi(\lambda x), x-\lambda x\rangle & =\langle w(\|x\|) x-w(\|\lambda x\|) \lambda x,(1-\lambda) x\rangle \\
& =(w(\|x\|)\|x\|-w(\|\lambda x\|)\|\lambda x\|)(1-\lambda)\|x\| .
\end{aligned}
$$

Using the fact that $t \mapsto w(t) t$ is strictly increasing, one can easily show that

$$
[w(\|x\|)\|x\|-w(\|\lambda x\|)\|\lambda x\|](1-\lambda)>0, \quad \forall \lambda \geq 0, \lambda \neq 1 .
$$

Consider now any $x, y \in \mathbb{R}^{N}, x \neq y$. We have

$$
\begin{aligned}
\langle\phi(x)-\phi(y), x-y\rangle & =w(\|x\|)\|x\|^{2}+w(\|y\|)\|y\|^{2}-(w(\|x\|)+w(\|y\|))\langle x, y\rangle \\
& \geq w(\|x\|)\|x\|^{2}+w(\|y\|)\|y\|^{2}-(w(\|x\|)+w(\|y\|))\|x\|\|y\| .
\end{aligned}
$$

Take $y_{1}=\lambda x$ such that $\left\|y_{1}\right\|=\|y\|$, with $\lambda \geq 0$. It follows that

$$
\begin{aligned}
& w(\|x\|)\|x\|^{2}+w(\|y\|)\|y\|^{2}-(w(\|x\|)+w(\|y\|))\|x\|\|y\| \\
& =w(\|x\|)\|x\|^{2}+w\left(\left\|y_{1}\right\|\right)\left\|y_{1}\right\|^{2}-\left(w(\|x\|)+w\left(\left\|y_{1}\right\|\right)\right)\|x\|\left\|y_{1}\right\| \\
& =\left\langle\phi(x)-\phi\left(y_{1}\right), x-y_{1}\right\rangle>0
\end{aligned}
$$

(the last inequality holds by the first case). Then the claim follows.

Coming back to problem (2.1), if a $C^{1}$ function $u: I \rightarrow \mathbb{R}^{N}$ solves the equation $\left(\phi\left(u^{\prime}\right)\right)^{\prime}=h(t)$, of course there exists $a \in \mathbb{R}^{N}$ such that

$$
\phi\left(u^{\prime}(t)\right)=a+H(h)(t)
$$

where $H$ is the integral operator

$$
H(h)(t)=\int_{0}^{t} h(s) d s
$$

Remark 2.2. Notice that the condition $u^{\prime}(0)=u^{\prime}(T)$ implies that $\int_{0}^{T} h(t) d t=0$ and this justifies the assumption that $h \in L_{m}^{1}$.

By the inversion of $\phi$ in $(2.2)$, we have

$$
u^{\prime}(t)=\phi^{-1}(a+H(h)(t)),
$$

and thus the image of $H(h)$, which contains the origin of $\mathbb{R}^{N}$, is included in an open ball with radius 1 . Call $\widetilde{D}$ the set of functions $h$ in $L_{m}^{1}$ such that there exists $a \in \mathbb{R}^{N}$ with

$$
\|a+H(h)(t)\|<1, \quad \forall t \in I .
$$


The set $\widetilde{D}$ is unbounded in $L_{m}^{1}$. Indeed, take for simplicity $T=1$ and consider the sequence of real functions $\left\{h_{n}\right\}_{n \in \mathbb{N}}$, where

$$
h_{n}:[0,1] \rightarrow \mathbb{R}, \quad h_{n}(t)= \begin{cases}n & t \in[k / n,(2 k+1) /(2 n)) \\ -n & t \in[(2 k+1) /(2 n),(k+1) / n) \cup\{1\},\end{cases}
$$

$k=0, \ldots, n-1$. Consider the sequence $\left\{k_{n}\right\} \subseteq L_{m}^{1}$, where $k_{n}=\left(h_{n}, 0, \ldots, 0\right)$. A straightforward computation shows that, for each $n$,

$$
\left\|k_{n}\right\|_{L^{1}}=n, \quad \text { and } \quad\left\|H\left(k_{n}\right)\right\|_{0}=1 / 2,
$$

that is, $\left\{k_{n}\right\}$ is an unbounded sequence contained in $\widetilde{D}$.

Moreover $\widetilde{D}$ is open in $L_{m}^{1}$. To see this, let $h \in \widetilde{D}$ be given. Suppose, without loss of generality, that $\|H(h)(t)\|<1$, for each $t \in I$. Given any $\varepsilon$ in $L_{m}^{1}$, one has

$$
\|H(h+\varepsilon)\|_{0} \leq\|H(h)\|_{0}+\|H(\varepsilon)\|_{0} \leq\|H(h)\|_{0}+\|\varepsilon\|_{L^{1}} .
$$

Therefore $\widetilde{D}$ contains the open ball in $L_{m}^{1}$ of center $h$ and radius $1-\|H(h)\|_{0}$ and the claim follows.

The open ball of $L_{m}^{1}$ of center zero and radius 2 is contained in $\widetilde{D}$. To see this, consider first any map $g \in L^{1}(I, \mathbb{R})$ such that $\int_{0}^{T} g(t) d t=0$. Then, define

$$
g_{+}(t)=\left\{\begin{array}{ll}
g(t) & \text { if } g(t) \geq 0 \\
0 & \text { if } g(t)<0
\end{array} \quad \text { and } \quad g_{-}(t)= \begin{cases}0 & \text { if } g(t) \geq 0 \\
-g(t) & \text { if } g(t)<0 .\end{cases}\right.
$$

As $\int_{0}^{T} g(t) d t=0$, one has that $\left\|g_{+}\right\|_{L^{1}}=\left\|g_{-}\right\|_{L^{1}}=\frac{1}{2}\|g\|_{L^{1}}$. In addition, one has

$$
\left|\int_{0}^{t} g(s) d s\right| \leq\left\|g_{+}\right\|_{L^{1}}, \quad \forall t \in I .
$$

Hence

$$
\|H(g)\|_{0} \leq \frac{1}{2}\|g\|_{L^{1}}
$$

Now consider any $h=\left(h_{1}, \ldots, h_{N}\right) \in L_{m}^{1}$, with $\|h\|_{L^{1}}<2$. It is immediate to check that

$$
\|H(h)\|_{0} \leq \frac{1}{2}\|h\|_{L^{1}}<1
$$

and this proves the assert.

Coming back to problem (2.1), we have seen that it admits a solution only if $h$ belongs to $\widetilde{D}$. Then, any $C^{1}$ solution $u$ can be written as

$$
u(t)=u(0)+\int_{0}^{t} \phi^{-1}(a+H(h)(s)) d s .
$$

The boundary condition $u(0)=u(T)$ implies that

$$
\int_{0}^{T} \phi^{-1}(a+H(h)(t)) d t=0 .
$$

Therefore problem (2.1) admits a solution in $\mathcal{C}_{T}^{1}$ if and only if $h$ belongs to the subset $D$ of $\widetilde{D}$ defined as the set of functions $h \in \widetilde{D}$ such that there exists $a \in \mathbb{R}^{N}$ verifying (2.4). The following proposition lists some properties of $D$.

Proposition 2.3. The following conditions hold. 
(1) For any $h \in D$ the point $a \in \mathbb{R}^{N}$ such that

$$
\int_{0}^{T} \phi^{-1}(a+H(h)(t)) d t=0
$$

is unique and then defines a map $\alpha: D \rightarrow \mathbb{R}^{N}$ which is bounded and continuous.

(2) The set $D$ is open, unbounded in $L_{m}^{1}$, and contains the open ball in $L_{m}^{1}$ with center zero and radius $2 / 3$;

Proof. (1) Let $h \in D$ be given and consider the function

$$
G_{H(h)}(a)=\int_{0}^{T} \phi^{-1}(a+H(h)(t)) d t
$$

which is well defined and continuous on the set

$$
\left\{a \in \mathbb{R}^{N}:\|a+H(h)(t)\|<1 \forall t \in I\right\} .
$$

We have that

$$
\left\langle G_{H(h)}\left(a_{1}\right)-G_{H(h)}\left(a_{2}\right), a_{1}-a_{2}\right\rangle>0, \quad \text { if } a_{1} \neq a_{2} .
$$

Indeed,

$$
\begin{aligned}
& \left\langle G_{H(h)}\left(a_{1}\right)-G_{H(h)}\left(a_{2}\right), a_{1}-a_{2}\right\rangle= \\
& \int_{0}^{T}\left\langle\phi^{-1}\left(a_{1}+H(h)(t)\right)-\phi^{-1}\left(a_{2}+H(h)(t)\right), a_{1}+H(h)(t)-\left(a_{2}+H(h)(t)\right)\right\rangle d t>0 .
\end{aligned}
$$

The last inequality is a consequence of Lemma 2.1. Now (2.6) implies that $G_{H(h)}=0$ has a unique solution and thus it turns out well defined the map $\alpha: D \rightarrow \mathbb{R}^{N}$. Since $H(h)(0)=0$ for each $h \in D$, then $\alpha$ is bounded having image contained in the open ball in $\mathbb{R}^{N}$ with center zero and radius 1 .

To see the continuity of $\alpha$ we proceed as follows. Define the set

$$
C=\left\{l \in \mathcal{C}_{T, 0}: \exists a \in \mathbb{R}^{N} \text { with }\|a+l(t)\|<1, \forall t \in I, \text { and } \int_{0}^{T} \phi^{-1}(a+l(t)) d t=0\right\}
$$

and consider the function $\widetilde{\alpha}: C \rightarrow \mathbb{R}^{N}$, such that, for each $l \in C$,

$$
\int_{0}^{T} \phi^{-1}(\widetilde{\alpha}(l)+l(t)) d t=0 .
$$

Let us prove the continuity of $\widetilde{\alpha}$. Let $\left\{l_{n}\right\}$ be a sequence in $C$, converging to $l \in C$. Since $\widetilde{\alpha}$ is bounded, any subsequence of $\widetilde{\alpha}\left(l_{n}\right)$ admits a convergent subsequence, say $\widetilde{\alpha}\left(l_{n_{j}}\right) \rightarrow \widehat{a}$ as $j \rightarrow \infty$. Let us show that $\phi^{-1}(\widehat{a}+l(t))$ is well defined. To this purpose, denote $\bar{a}=\widetilde{\alpha}(l)$ and call $B$ an open ball centered at $\bar{a}$ such that $G_{l}$ is well defined on $\bar{B}$, where $G_{l}$ is given in (2.5). As seen for (2.6), Lemma 2.1 implies that $\left\langle G_{l}(a), a-\bar{a}\right\rangle>0$ for each $a \in \bar{B}, a \neq \bar{a}$. In particular

$$
\left\langle G_{l}(a), a-\bar{a}\right\rangle>0, \quad \forall a \in \partial B
$$

Observe that there exists a neighborhood $U$ of $l$ in $\mathcal{C}_{T, 0}$ such that, for each $x \in U, G_{x}$ is well defined on $\bar{B}$. In addition, the map

$$
x \mapsto \inf _{a \in \partial B}\left\langle G_{x}(a), a-\bar{a}\right\rangle,
$$

is easily seen to be continuous on $U$. Then

$$
\left\langle G_{m}(a), a-\bar{a}\right\rangle>0, \quad \forall a \in \partial B,
$$

for each function $m$ in a suitable neighborhood $V \subseteq U$ of $l$. This implies, by a simple application of the homotopy invariance property of the Brouwer degree, that the equation $G_{m}(a)=0$ has its (unique) 
solution in $\bar{B}$, given $m$ in $V$. Hence $\widetilde{\alpha}\left(l_{n_{j}}\right) \in \bar{B}$, for $j$ sufficiently large, and thus $\widehat{a}$ belongs to $\bar{B}$. Therefore $\phi^{-1}(\widehat{a}+l(t))$ is well defined. Now, by letting $j \rightarrow \infty$ in

$$
\int_{0}^{T} \phi^{-1}\left(\widetilde{\alpha}\left(l_{n_{j}}\right)+l_{n_{j}}(t)\right) d t=0
$$

we have that

$$
\int_{0}^{T} \phi^{-1}(\widehat{\alpha}+l(t)) d t=0
$$

and this proves the continuity of $\widetilde{\alpha}$. Finally, $\alpha=\widetilde{\alpha} \circ H$ and this shows the continuity of $\alpha$, being $H$ continuous.

(2) To prove that $D$ is open in $L_{m}^{1}$, we first observe that the set $C$, defined by (2.7), is open. Indeed, this can be proved by the same argument following inequality (2.8). Now, as $D=H^{-1}(C)$, we have that $D$ is open in $L_{m}^{1}$.

The unboundedness of $D$ can be proved in the same way as done for $\widetilde{D}$. Precisely, for simplicity let $T=1$, and take the sequence of real functions $\left\{h_{n}\right\}$, defined by formula (2.3). Then, let $\left\{k_{n}\right\} \subseteq L_{m}^{1}$ be given by $k_{n}=\left(h_{n}, 0, \ldots, 0\right), n \in \mathbb{N}$. For any $n$ the function

$$
G_{n}(a)=\int_{0}^{1} \phi^{-1}\left(a+H\left(k_{n}\right)(t)\right) d t
$$

is well defined, in particular, for any $a$ of the form $a=\left(a_{1}, 0, \ldots, 0\right)$, with $a_{1} \in(-1,1 / 2)$. Denote by $G_{n, j}, j=1, \ldots, N$, the $j$-th component of $G_{n}$. If $a$ is selected as above, we have that

$$
G_{n, j}(a)=0
$$

for any $a$ and any $j \geq 2$. In addition, $G_{n, 1}(a)>0$ if $a_{1} \geq 0$ and $G_{n, 1}(a)<0$ if $a_{1} \leq-1 / 2$. As $G_{n, 1}$ is continuous, it admits a zero for a suitable $a$. Therefore $\left\{k_{n}\right\} \subseteq D$, which turns out to be not bounded.

In order to show that $D$ contains the open ball in $L_{m}^{1}$ centered at zero with radius $2 / 3$ we first prove that the set $C$, defined by (2.7), contains the open ball in $\mathcal{C}_{T, 0}$ of center zero and radius $1 / 3$. Let $l \in \mathcal{C}_{T, 0}$, with $\|l\|_{0}<1 / 3$, be given. If $l$ is identically zero, then it clearly belongs to $C$. Thus, suppose that $l$ is not zero for some $t$. Denote $\delta=\|l\|_{0}$. Then consider $2 \delta<\delta^{\prime}<2 / 3$ and let $A$ be the closed ball in $\mathbb{R}^{N}$ with center zero and radius $\delta^{\prime}$. Observe that $\|a+l(t)\|<1$ for any $t \in I$ and any $a \in A$. We show now that

$$
\left\langle G_{l}(a), a\right\rangle>0, \quad \text { if }\|a\|=\delta^{\prime} .
$$

To this purpose denote $v:[0,1) \rightarrow \mathbb{R}$ the function such that $\phi^{-1}(x)=v(\|x\|) x$. We have

$$
\begin{aligned}
\left\langle G_{l}(a), a\right\rangle & =\int_{0}^{T}\left\langle\phi^{-1}(a+l(t)), a+l(t)\right\rangle d t-\int_{0}^{T}\left\langle\phi^{-1}(a+l(t)), l(t)\right\rangle d t \\
& \geq \int_{0}^{T} v(\|a+l(t)\|)\|a+l(t)\|^{2} d t-\int_{0}^{T} v(\|a+l(t)\|)\|a+l(t)\|\|l(t)\| d t \\
& =\int_{0}^{T} v(\|a+l(t)\|)\|a+l(t)\|(\|a+l(t)\|-\|l(t)\|) d t .
\end{aligned}
$$

The last integral turns out to be positive if we show that, given $a$ with $\|a\|=\delta^{\prime}$,

$$
\|a+l(t)\|>\|l(t)\|, \quad \forall t \in I .
$$

We have

$$
\|a+l(t)\|^{2} \geq\|a\|^{2}+\|l(t)\|^{2}-2\|a\|\|l(t)\| \geq\|l(t)\|^{2}
$$

because $\|a\|>2\|l(t)\|$ for each $t$. Hence the (2.10) holds and this proves the (2.9). Therefore, by an elementary topological degree argument, the equation $G_{l}(a)=0$ has a solution in $A$ and hence $l \in C$. Thus, $C$ contains the open ball in $\mathcal{C}_{T, 0}$ of center zero and radius $1 / 3$. 
Now, let $h=\left(h_{1}, \ldots, h_{N}\right) \in L_{m}^{1}$ with $\|h\|_{L^{1}}<2 / 3$. Define, for $i=1, \ldots, N$,

$$
h_{i}^{+}(t)=\left\{\begin{array}{ll}
h_{i}(t) & \text { if } h_{i}(t) \geq 0 \\
0 & \text { if } h_{i}(t)<0
\end{array} \quad \text { and } \quad h_{i}^{-}(t)= \begin{cases}0 & \text { if } h_{i}(t) \geq 0 \\
-h_{i}(t) & \text { if } h_{i}(t)<0 .\end{cases}\right.
$$

As $\int_{0}^{T} h(t) d t=0$, one has that, for any $i,\left\|h_{i}^{+}\right\|_{L^{1}}=\left\|h_{i}^{-}\right\|_{L^{1}}=\frac{1}{2}\left\|h_{i}\right\|_{L^{1}}$ and thus

$$
\|h\|_{L^{1}}=2\left(\sum_{i=1}^{N}\left\|h_{i}\right\|_{L_{1}}^{2}\right)^{1 / 2} .
$$

In addition,

$$
\left|\int_{0}^{t} h_{i}(t) d t\right| \leq\left\|h_{i}^{+}\right\|_{L^{1}}, \quad \forall t \in I, i=1, \ldots N
$$

Then

$$
2\|H(h)(t)\|_{\mathbb{R}^{N}} \leq\|h\|_{L^{1}}, \quad \forall t \in I,
$$

and, finally,

$$
2\|H(h)\|_{0} \leq\|h\|_{L^{1}} .
$$

This proves that $D$ contains the open ball in $L_{m}^{1}$ with center zero and radius $2 / 3$.

We have seen that problem (2.1) has a solution if and only if $h \in D$. Actually, for any $h \in D$, we have infinite solutions which differ by a constant and can be written as

$$
u(t)=u(0)+H\left(\phi^{-1}[\alpha(h)+H(h)]\right)(t),
$$

where, by an abuse of notation, $\phi^{-1}[\alpha(h)+H(h)]$ is the continuous map $t \mapsto \phi^{-1}[\alpha(h)+H(h)(t)]$.

Define $P: \mathcal{C}_{T}^{1} \rightarrow \mathcal{C}_{T}^{1}$ as $P u=u(0)$. Observe that $\mathcal{C}_{T}^{1}$ admits the splitting

$$
\mathcal{C}_{T}^{1}=E_{1} \oplus E_{2},
$$

where $E_{1}$ contains the maps $\widetilde{u}$ such that $\widetilde{u}(0)=0$ and $E_{2}$ is the $N$-dimensional subspace of constant maps. It is immediate to see that $P$ is the continuous projection onto $E_{2}$ by the above decomposition. In addition consider $Q: L^{1} \rightarrow L^{1}$, defined as $Q h=\frac{1}{T} \int_{0}^{T} h(t) d t$. One can split $L^{1}$ as

$$
L^{1}=L_{m}^{1} \oplus F_{2},
$$

where $F_{2}$ is the $N$-dimensional subspace of constant maps ${ }^{1}$. The operator $Q$ turns easily out to be the continuous projection on $F_{2}$ with the above splitting of $L^{1}$. Then, consider the subset $\widehat{D}$ of $L^{1}$, given by

$$
\widehat{D}=D+F_{2},
$$

and the nonlinear operator $K: \widehat{D} \rightarrow \mathcal{C}_{T}^{1}$, defined as

$$
K(\widehat{h})(t)=H\left(\phi^{-1}[\alpha((I-Q) \widehat{h})+H((I-Q) \widehat{h})]\right)(t) .
$$

If a $C^{1}$ function $u$ is a solution of (2.1), for a given $h \in D$, of course $u$ solves the equation

$$
u=P u+Q h+K(h) .
$$

\footnotetext{
${ }^{1}$ The reader could notice that $E_{2}$ and $F_{2}$ are actually different, being contained in different Banach spaces.
} 
Conversely, if $u \in \mathcal{C}_{T}^{1}$ is a solution of (2.13), for a given $h \in \widehat{D}$, it follows that $h$ belongs to $D$ and $u$ solves (2.1). The idea of studying equation (2.13), in order to find a solution of (2.1), is particularly important if we consider an abstract periodic problem

$$
\left\{\begin{array}{l}
\left(\phi\left(u^{\prime}\right)\right)^{\prime}=G(u)(t) \\
u(0)=u(T) \\
u^{\prime}(0)=u^{\prime}(T),
\end{array}\right.
$$

where $G: \mathcal{C}^{1} \rightarrow \widehat{D}$ can be supposed continuous. In fact, if we define $\mathcal{G}: \mathcal{C}_{T}^{1} \rightarrow \mathcal{C}_{T}^{1}$ by

$$
\mathcal{G}(u)=P u+Q G(u)+K(G(u)),
$$

we observe that problem (2.14) is equivalent to the fixed point problem

$$
u=\mathcal{G}(u),
$$

which can be studied, under suitable conditions, by topological methods. Following this idea, in the next section we will apply the Leray-Schauder degree to obtain our main result, that is, as said in the Introduction, an existence theorem for the problem

$$
\left\{\begin{array}{l}
\left(\phi\left(u^{\prime}\right)\right)^{\prime}=f\left(t, u, u^{\prime}\right) \\
u(0)=u(T) \\
u^{\prime}(0)=u^{\prime}(T)
\end{array}\right.
$$

where $\phi$ is as above and $f: I \times \mathbb{R}^{n} \times \mathbb{R}^{N} \rightarrow \mathbb{R}^{N}$ is a Carathéodory function.

We conclude this section by proving crucial properties of $K$.

Proposition 2.4. The map $K$ is continuous and sends equi-integrable sets of $\widehat{D}$ into relatively compact sets in $\mathcal{C}_{T}^{1}$.

Proof. The continuity of $K$ as valued in $\mathcal{C}$ is a straightforward consequence of the fact that this map is a composition of continuous maps. In addition

$$
(K(\widehat{h}))^{\prime}(t)=\phi^{-1}[\alpha((I-Q) \widehat{h})+H((I-Q) \widehat{h})](t) .
$$

That is, $K^{\prime}$ is a composition of continuous operators and thus $K$ is continuous. Consider an equi-integrable set $S$ of $L^{1}$, contained in $\widehat{D}$, and let $g \in L^{1}(I, \mathbb{R})$ be such that, for all $h \in S$,

$$
\|h(t)\| \leq g(t) \quad \text { a.e. in } I \text {. }
$$

Let us show that $\overline{K(S)}$ is compact. To see this consider first a sequence $\left\{k_{n}\right\}$ of $K(S)$ and let $\left\{h_{n}\right\}$ be such that $K\left(h_{n}\right)=k_{n}$. For any $t_{1}, t_{2} \in I$ we have

$$
\begin{aligned}
\left\|H(I-Q)\left(h_{n}\right)\left(t_{1}\right)-H(I-Q)\left(h_{n}\right)\left(t_{2}\right)\right\| & \leq|| \int_{t_{2}}^{t_{1}} h_{n}(s) d s||+|| Q h_{n} \|\left|t_{1}-t_{2}\right| \\
& \leq\left|\int_{t_{2}}^{t_{1}} g(s) d s\right|+\frac{\left|t_{1}-t_{2}\right|}{T} \int_{0}^{T} g(s) d s .
\end{aligned}
$$

Therefore the sequence $\left\{H(I-Q)\left(h_{n}\right)\right\}$ is bounded and equicontinuous and then, by Ascoli-Arzelà Theorem, it admits a convergent subsequence in $\mathcal{C}$, say $\left\{H(I-Q)\left(h_{n_{j}}\right)\right\}$. Up to a subsequence, $\{\alpha((I-$ $\left.Q)\left(h_{n_{j}}\right)\right)+H\left((I-Q)\left(h_{n_{j}}\right)\right\}$ converges in $\mathcal{C}$. In addition we have that

$$
\left(K\left(h_{n_{j}}\right)\right)^{\prime}(t)=\phi^{-1}\left[\left\{\alpha\left((I-Q)\left(h_{n_{j}}\right)\right)+H\left((I-Q)\left(h_{n_{j}}\right)\right\}\right](t)\right.
$$

and, by the continuity of $\phi^{-1},\left(K\left(h_{n_{j}}\right)\right)^{\prime}$ is convergent in $\mathcal{C}$. Therefore $\left\{k_{n_{j}}\right\}=\left\{K\left(h_{n_{j}}\right)\right\}$ converges in $\mathcal{C}_{T}^{1}$. Now consider a sequence $\left\{k_{n}\right\}$ belonging to $\overline{K(S)}$ (that is, not necessarily to $\left.K(S)\right)$. Let $\left\{l_{n}\right\} \subseteq K(S)$ be such that $\left\|l_{n}-k_{n}\right\|_{1} \rightarrow 0$ as $n \rightarrow \infty$. Let in addition $\left\{l_{n_{j}}\right\}$ be a subsequence of $\left\{l_{n}\right\}$ that converges to $l$. Therefore, $l \in \overline{K(S)}$ and $\left\{k_{n_{j}}\right\} \rightarrow l$, and this completes the proof. 


\section{MAIN RESULt}

In this section we present the main result of this paper, that is, an existence theorem for the periodic boundary value problem

$$
\left\{\begin{array}{l}
\left(\phi\left(u^{\prime}\right)\right)^{\prime}=f\left(t, u, u^{\prime}\right) \\
u(0)=u(T) \\
u^{\prime}(0)=u^{\prime}(T)
\end{array}\right.
$$

where $\phi$ is as in the above section and $f: I \times \mathbb{R}^{N} \times \mathbb{R}^{N} \rightarrow \mathbb{R}^{N}$ is a Carathéodory function, that is,

i) for almost every $t \in I, f(t, \cdot, \cdot)$ is continuous;

ii) for any $(x, y) \in \mathbb{R}^{N} \times \mathbb{R}^{N}, f(\cdot, x, y)$ is measurable;

iii) for any $\rho>0$ there exists $g \in L^{1}(I, \mathbb{R})$ such that, for almost every $t \in I$ and every $(x, y) \in \mathbb{R}^{N} \times \mathbb{R}^{N}$, with $\|x\| \leq \rho$ and $\|y\| \leq \rho$, we have

$$
\|f(t, x, y)\| \leq g(t) .
$$

Theorem 3.1. Let $\Omega$ be a bounded open subset of $\mathcal{C}_{T}^{1}$ such that the following conditions hold:

(1) for any $u \in \bar{\Omega}$ the map $t \mapsto f\left(t, u(t), u^{\prime}(t)\right)$ belongs to $\widehat{D}$, where $\widehat{D}$ is defined by (2.12);

(2) for each $\lambda \in(0,1)$ the problem

$$
\left\{\begin{array}{l}
\left(\phi\left(u^{\prime}\right)\right)^{\prime}=\lambda f\left(t, u, u^{\prime}\right) \\
u(0)=u(T) \\
u^{\prime}(0)=u^{\prime}(T),
\end{array}\right.
$$

has no solution on $\partial \Omega$;

(3) the equation

$$
F(a):=\int_{0}^{T} f(t, a, 0) d t=0
$$

has no solution on $\partial \Omega_{2}$, where $\Omega_{2}:=\Omega \cap E_{2}$ and $E_{2}$ is the subspace of $\mathcal{C}_{T}^{1}$ in the splitting (2.11);

(4) the Brouwer degree

is well defined and nonzero.

$$
\operatorname{deg}_{B}\left(F, \Omega_{2}, 0\right)
$$

Then problem (3.1) has a solution in $\Omega$.

Proof. Let $N_{f}$ denote the Nemytski operator associated to $f$, that is,

$$
N_{f}: \mathcal{C}_{T}^{1} \rightarrow L^{1}, \quad N_{f}(u)(t)=f\left(t, u(t), u^{\prime}(t)\right) .
$$

Consider the problem

$$
\left\{\begin{array}{l}
\left(\phi\left(u^{\prime}\right)\right)^{\prime}=\lambda N_{f}(u)+(1-\lambda) Q N_{f}(u) \\
u(0)=u(T) \\
u^{\prime}(0)=u^{\prime}(T) .
\end{array}\right.
$$

For $\lambda \in(0,1]$, if $u$ is a solution of $(3.2)$, then, as seen in the previous section, condition $u^{\prime}(0)=u^{\prime}(T)$ implies $Q N_{f}(u)=0$ and hence $u$ solves problem (3.4) as well. Conversely, if $u$ is a solution of problem (3.4), then $Q N_{f}(u)=0$ since it is easy to see that

$$
Q\left[\lambda N_{f}(u)+(1-\lambda) Q N_{f}(u)\right]=Q N_{f}(u)
$$

and thus $u$ solves problem $(3.2)$ ( $\lambda$ still belongs to $(0,1]$ ). Let us now consider problem (3.4). It can be written in the equivalent form

$$
u=\mathcal{K}(u, \lambda)
$$

where

$$
\begin{aligned}
\mathcal{K}(u, \lambda) & =P u+Q N_{f}(u)+\left(K \circ\left[\lambda N_{f}+(1-\lambda) Q N_{f}\right]\right)(u) \\
& =P u+Q N_{f}(u)+\left(K \circ\left[\lambda(I-Q) N_{f}\right]\right)(u)
\end{aligned}
$$


is well defined in $\bar{\Omega} \times[0,1]$. Suppose that (3.4) has no solution on $\partial \Omega$ for $\lambda=1$, since, otherwise, the theorem is proved. Take $\lambda=0$. Problem (3.4) becomes

$$
\left\{\begin{array}{l}
\left(\phi\left(u^{\prime}\right)\right)^{\prime}=\frac{1}{T} \int_{0}^{T} f\left(t, u(t), u^{\prime}(t)\right) d t \\
u(0)=u(T) \\
u^{\prime}(0)=u^{\prime}(T) .
\end{array}\right.
$$

It follows that $\int_{0}^{T} f\left(t, u(t), u^{\prime}(t)\right) d t=0$ and this implies that $u$ is a constant function, say $u(t)=c$. Therefore, we have

$$
\int_{0}^{T} f(t, c, 0) d t=0
$$

By assumption (2), $c \notin \partial \Omega_{2}$. Therefore we obtain that the equation

$$
u-\mathcal{K}(u, \lambda)=0
$$

has no solution on $\partial \Omega \times[0,1]$. In addition, as $f$ is Carathéodory, the nonlinear map $\mathcal{N}: \mathcal{C}_{T}^{1} \times[0,1] \rightarrow L^{1}$, defined by

$$
\mathcal{N}(u, \lambda)=\lambda N_{f}(u)+(1-\lambda) Q N_{f}(u)
$$

is continuous and takes bounded sets into equi-integrable sets. This implies that, recalling Proposition $2.4, \mathcal{K}$ is completely continuous. We can apply the homotopy invariance property of the Leray-Schauder degree to the map $(u, \lambda) \mapsto u-\mathcal{K}(u, \lambda)$, obtaining

$$
\operatorname{deg}_{L S}(I-\mathcal{K}(\cdot, 0), \Omega, 0)=\operatorname{deg}_{L S}(I-\mathcal{K}(\cdot, 1), \Omega, 0) .
$$

We can now say that problem $(3.1)$ has a solution in $\bar{\Omega}$ if we prove that $\operatorname{deg}_{L S}(I-\mathcal{K}(\cdot, 0), \Omega, 0) \neq 0$. To see this we apply a finite-dimensional reduction property of the Leray-Schauder degree, associated with assumption (3). Observe first that $K(0)=0$, then

$$
\mathcal{K}(u, 0)=P u+Q N_{f}(u) .
$$

To compare the Leray-Schauder degree of the triple $(I-\mathcal{K}(\cdot, 0), \Omega, 0)$ with the Brouwer degree of $\left(F, \Omega_{2}, 0\right)$, consider the splitting $(2.11)$ of $\mathcal{C}_{T}^{1}$. The operator $I-\mathcal{K}(\cdot, 0)$ can be represented in block-matrix form as

$$
I-\mathcal{K}(\cdot, 0)=\left(\begin{array}{ll}
I_{E_{1}} & -\mathcal{K}_{12} \\
0 & -F
\end{array}\right) .
$$

By the properties of the Leray-Schauder degree we have that

$$
\operatorname{deg}_{L S}(I-\mathcal{K}(\cdot, 0), \Omega, 0)=(-1)^{N} \operatorname{deg}_{B}\left(F, \Omega_{2}, 0\right)
$$

and this completes the proof.

\section{An Application}

In this section we show an application of Theorem 3.1 to the two-dimensional problem

$$
\left\{\begin{array}{l}
\left(\frac{u_{1}^{\prime}}{\sqrt{1+\left|u^{\prime}\right|^{2}}}\right)^{\prime}=g_{1}(t)\left(u_{1}^{5}+\left(u_{1}^{\prime}\right)^{6}\right)+\eta_{1}, \\
\left(\frac{u_{2}^{\prime}}{\sqrt{1+\left|u^{\prime}\right|^{2}}}\right)^{\prime}=g_{2}(t)\left(u_{2}^{5}+\left(u_{2}^{\prime}\right)^{6}\right)+\eta_{2}, \\
u_{1}(0)=u_{1}(1), \quad u_{1}^{\prime}(0)=u_{1}^{\prime}(1) \\
u_{2}(0)=u_{2}(1), \quad u_{2}^{\prime}(0)=u_{2}^{\prime}(1)
\end{array}\right.
$$

where $g_{1}, g_{2}$ are continuous real functions on $[0,1]$ such that $1 / 2 \leq g_{i}(t) \leq 1$, for each $t \in[0,1], i=1,2$, and $\eta_{1}, \eta_{2}$ are real constants. Our purpose is to prove that, for $\eta_{1}, \eta_{2}$ sufficiently small, (4.1) admits a solution in the closed ball $\bar{\Omega}$ of $\mathcal{C}_{T}^{1}(T=1)$ with center zero and radius $1 / 2$. 
Remark 4.1. Recalling Remark 1.1, if $u \in \mathcal{C}_{T}^{1}$ solves system $(4.1), \phi\left(u^{\prime}\right)$ is absolutely continuous ( $\phi$ being defined as $\left.\phi(t)=t / \sqrt{1+t^{2}}\right)$. It is immediate to verify that $u^{\prime}$ is absolutely continuous as well. Now, observe that $u^{\prime \prime}$ coincides a.e. with a continuous function and thus it can be continuously extended to $[0,1]$. This implies that $u^{\prime}$ is actually $C^{1}$ and then any solution of the problem is actually a $C^{2}$ function.

We start by showing that (4.1) has no solution on $\partial \Omega$ for $\eta_{1}, \eta_{2}$ in a suitable neighborhood of zero. Let $u \in \partial \Omega$ be given, that is,

We consider different cases.

$$
\|u\|_{1}=\|u\|_{0}+\left\|u^{\prime}\right\|_{0}=1 / 2
$$

(i) Suppose $2 / 5 \leq\|u\|_{0} \leq 1 / 2$. Thus, for some $t, u_{1}^{2}(t)+u_{2}^{2}(t) \geq 4 / 25$. We can suppose, without loss of generality, that $\left|u_{1}(t)\right| \geq \sqrt{2} / 5$, for some $t$. Since $\|u\|_{0} \geq 2 / 5$, we have $\left\|u^{\prime}\right\|_{0} \leq 1 / 10$, and then $\left|u_{1}^{\prime}(t)\right| \leq 1 / 10$ for every $t \in[0,1]$. By the mean value theorem, $|u(t)| \geq(2 \sqrt{2}-1) / 10$ for each $t \in[0,1]$. In particular, $u_{1}$ has constant sign.

If $u_{1}(t)$ is positive for any $t$, a computation shows that

$$
g_{1}(t)\left(u_{1}^{5}+\left(u_{1}^{\prime}\right)^{6}\right)+\eta_{1}>0, \quad \forall t \in[0,1]
$$

if $\eta_{1}>-10^{-4}$. If otherwise $u_{1}(t)$ is negative for any $t$,

for $\eta_{1}<10^{-4}$.

$$
g_{1}(t)\left(u_{1}^{5}+\left(u_{1}^{\prime}\right)^{6}\right)+\eta_{1}<0, \quad \forall t \in[0,1]
$$

By the boundary conditions, $u_{1}^{\prime} / \sqrt{1+\left|u^{\prime}\right|^{2}}$ cannot be strictly monotone on $[0,1]$. That is, the first equation in (4.1) has no solution on $\partial \Omega$ satisfying $2 / 5 \leq\|u\|_{0} \leq 1 / 2$, if $\eta_{1} \in\left(-10^{-4}, 10^{-4}\right)$. Generalizing this argument, problem (4.1) has no solution on $\partial \Omega$ with $2 / 5 \leq\|u\|_{0} \leq 1 / 2$, if $\eta_{1}, \eta_{2} \in\left(-10^{-4}, 10^{-4}\right)$.

(ii) Suppose $1 / 4 \leq\|u\|_{0}<2 / 5$. This implies $1 / 10<\left\|u^{\prime}\right\|_{0} \leq 1 / 4$ and hence $\left|u_{1}^{\prime}(t)\right|$ and $\left|u_{2}^{\prime}(t)\right|$ are $\leq 1 / 4$ for any $t$.

The two equations of (4.1) can be written in the following equivalent way:

$$
\left\{\begin{array}{l}
u_{1}^{\prime \prime}\left(1+\left(u_{2}^{\prime}\right)^{2}\right)-u_{1}^{\prime} u_{2}^{\prime} u_{2}^{\prime \prime}=\left(1+\left|u^{\prime}\right|^{2}\right)^{3 / 2}\left[g_{1}(t)\left(u_{1}^{5}+\left(u_{1}^{\prime}\right)^{6}\right)+\eta_{1}\right] \\
u_{2}^{\prime \prime}\left(1+\left(u_{1}^{\prime}\right)^{2}\right)-u_{1}^{\prime} u_{2}^{\prime} u_{1}^{\prime \prime}=\left(1+\left|u^{\prime}\right|^{2}\right)^{3 / 2}\left[g_{2}(t)\left(u_{2}^{5}+\left(u_{2}^{\prime}\right)^{6}\right)+\eta_{2}\right] .
\end{array}\right.
$$

Since $\left\|u^{\prime}\right\|_{0} \geq 1 / 10$, then $\left(u_{1}^{\prime}\right)^{2}(t)+\left(u_{2}^{\prime}\right)^{2}(t) \geq 1 / 100$ for some $t$. Suppose, without loss of generality, that $\left|u_{1}^{\prime}(t)\right| \geq \sqrt{2} / 20$ for some $t$. By the boundary condition on $u_{1}^{\prime}$, there exists $t^{\prime} \in[0,1]$ such that $u_{1}^{\prime}\left(t^{\prime}\right)=0$. This implies that there exists $t^{\prime \prime} \in[0,1]$ such that $\left|u_{1}^{\prime \prime}\left(t^{\prime \prime}\right)\right| \geq \sqrt{2} / 20$.

Now, a computation shows that, if

$$
\left|u_{1}^{\prime \prime}\left(t^{\prime \prime}\right)\left(1+\left(u_{2}^{\prime}\right)^{2}\left(t^{\prime \prime}\right)\right)-u_{1}^{\prime}\left(t^{\prime \prime}\right) u_{2}^{\prime}\left(t^{\prime \prime}\right) u_{2}^{\prime \prime}\left(t^{\prime \prime}\right)\right|<0.05
$$

then

$$
\left|u_{2}^{\prime \prime}\left(t^{\prime \prime}\right)\left(1+\left(u_{1}^{\prime}\right)^{2}\left(t^{\prime \prime}\right)\right)-u_{1}^{\prime}\left(t^{\prime \prime}\right) u_{2}^{\prime}\left(t^{\prime \prime}\right) u_{1}^{\prime \prime}\left(t^{\prime \prime}\right)\right|>0.05
$$

and this a consequence of the inequality

$$
\left|u_{1}^{\prime}(t) u_{2}^{\prime}(t)\right|<1 / 32, \quad \forall t \in[0,1] .
$$

Choosing $\eta_{1}, \eta_{2} \in\left(-10^{-4}, 10^{-4}\right)$, we obtain that, for any $t \in[0,1]$,

$$
\left(1+\left|u^{\prime}\right|^{2}\right)^{3 / 2}\left|g_{1}(t)\left(u_{1}^{5}+\left(u_{1}^{\prime}\right)^{6}\right)+\eta_{1}\right| \leq 0.05,
$$

and

$$
\left(1+\left|u^{\prime}\right|^{2}\right)^{3 / 2}\left|g_{2}(t)\left(u_{2}^{5}+\left(u_{2}^{\prime}\right)^{6}\right)+\eta_{2}\right| \leq 0.05 .
$$

We conclude system (4.1) has no solution on $\partial \Omega$ if $1 / 4 \leq\|u\|_{0} \leq 2 / 5$, with $\eta_{1}, \eta_{2} \in\left(-10^{-4}, 10^{-4}\right)$. 
(3) As a final step, suppose $\|u\|_{0}<1 / 4$. This implies $\left\|u^{\prime}\right\|_{0}>1 / 4$. In addition, $\left|u_{1}^{\prime}(t)\right|$ and $\left|u_{2}^{\prime}(t)\right|$ are $\leq 1 / 2$ for any $t$. We proceed as in the above step. For some $t,\left(u_{1}^{\prime}\right)^{2}(t)+\left(u_{2}^{\prime}\right)^{2}(t)>1 / 16$, and, without loss of generality, assume that $\left|u_{1}^{\prime}(t)\right|>\sqrt{2} / 8$. Analogously to the previous case, there exists $t^{\prime \prime}$ such that $\left|u_{1}^{\prime \prime}\left(t^{\prime \prime}\right)\right|>\sqrt{2} / 8$.

Choosing $\eta_{1}, \eta_{2} \in\left(-10^{-4}, 10^{-4}\right)$, we obtain that, for any $t \in[0,1]$,

$$
\left(1+\left|u^{\prime}\right|^{2}\right)^{3 / 2}\left|g_{1}(t)\left(u_{1}^{5}+\left(u_{1}^{\prime}\right)^{6}\right)+\eta_{1}\right| \leq 0.13,
$$

and

On the other hand, if

$$
\left(1+\left|u^{\prime}\right|^{2}\right)^{3 / 2}\left|g_{2}(t)\left(u_{2}^{5}+\left(u_{2}^{\prime}\right)^{6}\right)+\eta_{2}\right| \leq 0.13
$$

$$
\left|u_{1}^{\prime \prime}\left(t^{\prime \prime}\right)\left(1+\left(u_{2}^{\prime}\right)^{2}\left(t^{\prime \prime}\right)\right)-u_{1}^{\prime}\left(t^{\prime \prime}\right) u_{2}^{\prime}\left(t^{\prime \prime}\right) u_{2}^{\prime \prime}\left(t^{\prime \prime}\right)\right|<0.13
$$

then

$$
\left|u_{2}^{\prime \prime}\left(t^{\prime \prime}\right)\left(1+\left(u_{1}^{\prime}\right)^{2}\left(t^{\prime \prime}\right)\right)-u_{1}^{\prime}\left(t^{\prime \prime}\right) u_{2}^{\prime}\left(t^{\prime \prime}\right) u_{1}^{\prime \prime}\left(t^{\prime \prime}\right)\right|>0.13
$$

and this implies that there is no solution.

Summarizing this argument, problem (4.1) has no solution on $\partial \Omega$, with $\eta_{1}, \eta_{2} \in\left(-10^{-4}, 10^{-4}\right)$. Let us apply Theorem 3.1 to show that our problem has a solution in $\bar{\Omega}$. To this purpose, observe that the system

$$
\left\{\begin{array}{l}
\left(\frac{u_{1}^{\prime}}{\sqrt{1+\left|u^{\prime}\right|^{2}}}\right)^{\prime}=\lambda\left[g_{1}(t)\left(u_{1}^{5}+\left(u_{1}^{\prime}\right)^{6}\right)+\eta_{1}\right], \\
\left(\frac{u_{2}^{\prime}}{\sqrt{1+\left|u^{\prime}\right|^{2}}}\right)^{\prime}=\lambda\left[g_{2}(t)\left(u_{2}^{5}+\left(u_{2}^{\prime}\right)^{6}\right)+\eta_{2}\right], \\
u_{1}(0)=u_{1}(1), \quad u_{1}^{\prime}(0)=u_{1}^{\prime}(1), \\
u_{2}(0)=u_{2}(1), \quad u_{2}^{\prime}(0)=u_{2}^{\prime}(1),
\end{array}\right.
$$

has no solution for any $\lambda \in(0,1]$ and any $u \in \partial \Omega$, and this can be easily seen by the same argument used in the case when $\lambda=1$. Recalling points (3) and (4) in the statement of Theorem 3.1, the equation

$$
F(a, b)=\left(\int_{0}^{1} g_{1}(t) d t a^{5}+\eta_{1} d t \int_{0}^{1} g_{2}(t) d t b^{5}+\eta_{2} d t\right)=(0,0)
$$

has no solution on $\partial \Omega_{2}$, where $\Omega_{2}=(-1 / 2,1 / 2) \times(-1 / 2,1 / 2)$ for any given $\eta_{1}, \eta_{2} \in\left(-10^{-4}, 10^{-4}\right)$. It is immediate to see that

$$
\operatorname{deg}_{B}\left(F, \Omega_{2}, 0\right)=1 .
$$

Thus we can apply Theorem 3.1 to conclude that (4.1) admits a solution in $\Omega$ for $\eta_{1}, \eta_{2} \in\left(-10^{-4}, 10^{-4}\right)$. It is also immediate that any solution is nontrivial if $\eta_{1}$ and $\eta_{2}$ are not both zero.

\section{REFERENCES}

[1] P. Benevieri, J.M. do Ó and E.S. Medeiros, Periodic Solutions for Nonlinear Equations with Mean Curvature-like Operators, preprint.

[2] C. Bereanu and J. Mawhin, Nonlinear Neumann Boundary Value Problems with $\phi$-Laplacian Operators, to appear on Analele Univesitii Ovidius Constanta, Serie Matematica, special issue dedicated to D. Pascali, 2005.

[3] K. Deimling, Nonlinear Functional Analysis, Springer Verlag, Berlin, 1985.

[4] N. Lloyd, Degree Theory, Cambridge University press, 1978.

[5] R. Manásevich and J. Mawhin, Periodic Solutions for Nonlinear Systems with p-Laplacian-Like Operators, J. Differential Equations 145 (1998), 367-393.

[6] J. Mawhin, Periodic solutions of systems with $p$-Laplacian-like operators, Nonlinear analysis and its application to differential equations (Lisbon 1998), 37-63, Prog. Nonlinear Differential Equations Appl., 43, Birkhäuser, Boston, 2001. 
Pierluigi Benevieri, Dipartimento di Matematica Applicata "G. Sansone", Via S. Marta 3,I-50139 Firenze, ITALY.E-MAIL ADDRESS: PIERLUIGI.BENEVIERI@UNIFI.IT

João Marcos do Ó, Departamento de Matemática, Universidade federal da Paraiba, CCen, 58059-900 João Pessoa, PB, Brazil. E-mail address: JMbo@Mat.ufpb.Br

Everaldo Souto Medeiros, Departamento de Matemática, Universidade Federal da Paraiba, CCen, 58059900 João Pessoa, PB, Brazil. E-mail address: everaldo@mat.ufpb.BR 